\title{
Existence for Eventually Positive Solutions of High-Order Nonlinear Neutral Differential Equations with Distributed Delay
}

\author{
Huanhuan Zhao, ${ }^{1}$ Youjun Liu, ${ }^{1}$ and Jurang Yan $^{2}$ \\ ${ }^{1}$ College of Mathematics and Computer Sciences, Shanxi Datong University, Datong, \\ Shanxi 037009, China \\ 2 School of Mathematical Sciences, Shanxi University, Taiyuan, Shanxi 030006, China \\ Correspondence should be addressed to Huanhuan Zhao, zhh9791@126.com
}

Received 14 February 2012; Accepted 12 March 2012

Academic Editor: Mingshu Peng

Copyright (C) 2012 Huanhuan Zhao et al. This is an open access article distributed under the Creative Commons Attribution License, which permits unrestricted use, distribution, and reproduction in any medium, provided the original work is properly cited.

We consider the existence for eventually positive solutions of high-order nonlinear neutral differential equations with distributed delay. We use Lebesgue's dominated convergence theorem to obtain new necessary and sufficient condition for the existence of eventually positive solutions.

\section{Introduction and Preliminary}

In this paper, we consider the high-order nonlinear neutral differential equation:

$$
\left[r(t) x(t)-\int_{a}^{b} p(t, \tau) x(t-\tau) d \tau\right]^{(n)}+\int_{c}^{d} q(t, \sigma) f(x(t-\sigma)) d \sigma=0, \quad t \geq t_{0},
$$

and the associated inequality:

$$
\left[r(t) x(t)-\int_{a}^{b} p(t, \tau) x(t-\tau) d \tau\right]^{(n)}+\int_{c}^{d} q(t, \sigma) f(x(t-\sigma)) d \sigma \leq 0, \quad t \geq t_{0},
$$

(1) where $n$ is a positive integer, $0<a<b, 0<c<d$,

(2) $p \in C\left(\left[t_{0}, \infty\right) \times[a, b], R^{+}\right)$, 
(3) $r \in C\left(\left[t_{0}, \infty\right), R^{+}\right), r(t)>0, q \in C\left(\left[t_{0}, \infty\right) \times[c, d], R^{+}\right)$,

(4) $f(u)$ are continuously nondecreasing real function with respect to $u$ defined on $\mathrm{R}$ such that $u f(u)>0$, for $u>0$, for $n$ is positive odd integer; $f(u)$ are continuously decreasing real function with respect to $u$ defined on $R$ such that $u f(u)>0$, for $u>0$, for $n$ is positive even integer.

Recently, there has been a lot of activities concerning the existence of eventually positive solutions for nonlinear neutral differential equations. See [1-8]. In [1], Liu et al. have studied the even-order neutral differential equation:

$$
\left[a(t) x(t)-\sum_{i=1}^{m} b_{i}(t) x\left(t-\tau_{i}\right)\right]^{(n)}-\sum_{j=1}^{l} p_{j}(t) f_{j}\left(t, x\left(t-\sigma_{j}\right)\right)=0, \quad t \geq 0,
$$

and the associated differential inequality:

$$
\left[a(t) x(t)-\sum_{i=1}^{m} b_{i}(t) x\left(t-\tau_{i}\right)\right]^{(n)}-\sum_{j=1}^{l} p_{j}(t) f_{j}\left(t, x\left(t-\sigma_{j}\right)\right) \geq 0, \quad t \geq 0 .
$$

They have obtained that the existences of eventually positive solutions of (1.3) and (1.4) are equivalent. In [2], Ouyang et al. has studied the odd-order neutral differential equation:

$$
\left[a(t) x(t)-\sum_{i=1}^{m} b_{i}(t) x\left(t-\tau_{i}\right)\right]^{(n)}+\sum_{j=1}^{l} p_{j}(t) f_{j}\left(x\left(t-\sigma_{j}\right)\right)=0, \quad t \geq 0,
$$

and the associated differential inequality:

$$
\left[a(t) x(t)-\sum_{i=1}^{m} b_{i}(t) x\left(t-\tau_{i}\right)\right]^{(n)}+\sum_{j=1}^{l} p_{j}(t) f_{j}\left(x\left(t-\sigma_{j}\right)\right) \leq 0, \quad t \geq 0 .
$$

He has obtained that the existences of eventually positive solutions of (1.5) and (1.6) are equivalent.

As usual, a solution of (1.1) is a continuous function $x(t)$ defined on $[-\mu, \infty)$ such that $y(t):=r(t) x(t)-\int_{a}^{b} p(t, \tau) x(t-\tau) d \tau$ is $n$ times differentiable and (1.1) holds for all $n \geq 0$. Such a solution $x(t)$ is called an eventually positive solution if there is $T \geq t_{0}$, such that $x(t)>0$, for $t \geq T$. Here, $\mu=\max \{b, d\}$.

Lemma 1.1. Assume $\sup _{t \geq t_{0}} r(t)<\infty$, and $\int_{a}^{b} p(t, \tau) d \tau / r(t) \leq M$, let $x(t)$ is an eventually bounded positive solution of inequality (1.2), and set

$$
y(t)=r(t) x(t)-\int_{a}^{b} p(t, \tau) x(t-\tau) d \tau .
$$


If $n$ is a positive odd integer, then eventually,

$$
\begin{gathered}
y^{(n)}(t) \leq 0, \quad(-1)^{k+1} y^{(k)}(t)<0, \quad(k=0,1, \ldots, n-1), \\
\lim _{t \rightarrow \infty} y^{(k)}(t)=0, \quad(k=1,2, \ldots, n-1) .
\end{gathered}
$$

If $n$ is a positive even integer, then eventually,

$$
\begin{gathered}
y^{(n)}(t) \leq 0, \quad(-1)^{k} y^{(k)}(t)<0, \quad(k=0,1, \ldots, n-1), \\
\lim _{t \rightarrow \infty} y^{(k)}(t)=0, \quad(k=1,2, \ldots, n-1) .
\end{gathered}
$$

Proof. We have the following cases.

Case 1 (If $n$ is a positive odd integer). Because $x(t)$ is bounded, and $\sup _{t \geq t_{0}} r(t)<$ $\infty, \int_{a}^{b} p(t, \tau) d \tau / r(t) \leq M$, thus $y(t)$ is bounded. From (1.2), we have $y^{(n)}(t) \leq$ $-\int_{c}^{d} q(t, \sigma) f(x(t-\sigma)) d \sigma \leq 0$. Assume $y^{(n-1)}(t) \leq 0$, since $y^{(n)}(t) \leq 0, y^{(n-1)}(t)$ decreases, set $\lim _{t \rightarrow \infty} y^{(n-1)}(t)=L$, then $-\infty \leq L<0$.

Thus,

$$
y^{(n-2)}(t)-y^{(n-2)}\left(t_{0}\right)=\int_{t_{0}}^{t} y^{(n-1)}(t) d t \leq(L+\varepsilon)\left(t-t_{0}\right) \longrightarrow-\infty, \quad(t \longrightarrow \infty)
$$

that is, $\lim _{t \rightarrow \infty} y^{(n-2)}(t)=-\infty$. Simile, $\lim _{t \rightarrow \infty} y^{(k)}(t)=-\infty,(k=0,1, \ldots, n-2)$, this is a contradiction and $y(t)$ is bounded, therefore, $y^{(n-1)}(t)>0$.

Again, since it decreases, set $\lim _{t \rightarrow \infty} y^{(n-1)}(t)=L$, thus $L \geq 0$. Next, we proof $L=0$. Assume $L>0$, then,

$$
y^{(n-2)}(t)-y^{(n-2)}\left(t_{0}\right)=\int_{t_{0}}^{t} y^{(n-1)}(t) d t \geq(L-\varepsilon)\left(t-t_{0}\right) \longrightarrow \infty, \quad(t \longrightarrow \infty),
$$

then, $\lim _{t \rightarrow \infty} y^{(n-2)}(t)=\infty$. Simile, $\lim _{t \rightarrow \infty} y^{(k)}(t)=\infty,(k=0,1, \ldots, n-2)$, this is a contradiction and $y(t)$ is bounded, therefore, $L=0$. That is, $\lim _{t \rightarrow \infty} y^{(n-1)}(t)=0$. Similarly, we obtain

$$
(-1)^{k+1} y^{(k)}(t)<0, \quad(k=0,1, \ldots, n-1), \quad \lim _{t \rightarrow \infty} y^{(k)}(t)=0, \quad(k=1,2, \ldots, n-1) .
$$

Case 2 (If $n$ is a positive even integer). The proof of Case 2 is similar to that of part Case 1, therefore, it is omitted. We obtain

$$
(-1)^{k} y^{(k)}(t)<0, \quad(k=0,1, \ldots, n-1), \quad \lim _{t \rightarrow \infty} y^{(k)}(t)=0, \quad(k=1,2, \ldots, n-1)
$$

The proof is complete. 
Lemma 1.2 (see [3, page 21]). Let $\eta \in(-\infty, 0), \tau \in(0, \infty), t_{0} \in R$, and suppose that a function $x(t) \in C\left(\left[t_{0}-\tau, \infty\right), R\right)$ satisfies the inequality:

$$
x(t) \leq \eta+\max _{t-\tau \leq s \leq t} x(s), \quad t \geq t_{0} .
$$

Then, $x(t)$ cannot be a nonnegative function.

Lemma 1.3. Suppose that $\sup _{t \geq t_{0}} r(t)<\infty$, and $\int_{a}^{b} p(t, \tau) d \tau / a(t) \leq 1$. Let $x(t)$ be an eventually positive solution of (1.2) and set

$$
y(t)=r(t) x(t)-\int_{a}^{b} p(t, \tau) x(t-\tau) d \tau
$$

then eventually

$$
y(t)>0
$$

Proof. From (1.2) and (1.7), eventually, we have

$$
y^{(n)}(t) \leq-\int_{c}^{d} q(t, \sigma) f(x(t-\sigma)) d \sigma \leq 0,
$$

and the hypotheses on $q(t, \sigma), f(x)$, and $x(t)$ yield that $y^{(n)}$ is not eventually zero. Thus, $y^{(i)}(t)(i=0, l, \ldots, n-1)$ is eventually nonzero. Hence, if $y(t)>0$ does not hold, then eventually $y(t)<0, y^{\prime}(t)<0$, or $y(t)<0, y^{\prime}(t)>0$.

Case 1. $y(t)<0, y^{\prime}(t)<0$, then there exists $t_{1}>0$ such that $y(t) \leq y\left(t_{1}\right)<0$ for $t>t_{1}$. Then, $\alpha:=-y\left(t_{1}\right) / \sup _{t \geq t_{0}} r(t)>0$. In view of (1.3) and $\int_{a}^{b} p(t, \tau) d \tau / a(t) \leq 1$, we obtain

$$
\begin{aligned}
x(t) & =\frac{y(t)}{r(t)}+\frac{1}{r(t)} \int_{a}^{b} p(t, \tau) x(t-\tau) d \tau \\
& \leq-\alpha+\frac{1}{r(t)} \int_{a}^{b} p(t, \tau) d \tau \max _{t-b \leq s \leq t} x(s) \\
& \leq-\alpha+\max _{t-b \leq s \leq t} x(s) .
\end{aligned}
$$

According to Lemma 1.2, we obtain $x(t)<0$. This is a contradiction and so $y(t)$ is eventually positive.

Case 2. $y(t)<0, y^{\prime}(t)>0$, one argues that $y^{\prime \prime}(t)<0$ and repeats the argument to obtain that $y^{(n)}(t)>0$ which contradicts the offset after $y^{(n)}(t)<0$.

The proof is complete. 


\section{Comparison Theory of Existence for Eventually Positive Solution}

Theorem 2.1. Assume all conditions of Lemma 1.1 hold, $n$ is a positive odd integer. And

$\left(\mathrm{H}_{1}\right) p(t, \tau)+q(t, \sigma)>0$, for sufficiently large $t$.

Then (1.1) has an eventually bounded positive solution if and only if inequality (1.2) has an eventually bounded positive solution.

Proof. It is clear that an eventually bounded positive solution of (1.1) is also an eventually bounded positive solution of (1.2). So, it suffices to prove that if (1.2) has an eventually bounded positive solution $x(t)$, for $t>t_{0}$, then so does (1.1). Set

$$
y(t)=r(t) x(t)-\int_{a}^{b} p(t, \tau) x(t-\tau) d \tau
$$

It follows from Lemma 1.1 and (1.2) that eventually,

$$
\begin{gathered}
y^{(n)}(t) \leq 0, \quad(-1)^{k+1} y^{(k)}(t)>0, \quad(k=0,1, \ldots, n-1), \\
\lim _{t \rightarrow \infty} y^{k}(t)=0, \quad(k=1,2, \ldots, n-1) .
\end{gathered}
$$

By using (1.8) and integrating (1.2) from $t$ to $\infty$, we obtain

$$
\begin{aligned}
y^{(n-1)}(\infty)-y^{(n-1)}(t) & \leq-\int_{t}^{\infty}\left[\int_{c}^{d} q(t, \sigma) f(x(t-\sigma)) d \sigma\right] d s, \\
y^{(n-1)}(t) & \geq \int_{t}^{\infty}\left[\int_{c}^{d} q(s, \sigma) f(x(s-\sigma)) d \sigma\right] d s .
\end{aligned}
$$

By repeating the same procedure $n$ times and by using (1.8), we are led to the inequality:

$$
y(t) \geq \int_{t}^{\infty} d t_{n} \int_{t_{n}}^{\infty} d t_{n-1} \int_{t_{n-1}}^{\infty} d t_{n-2} \cdots \int_{t_{2}}^{\infty}\left[\int_{c}^{d} q(s, \sigma) f(x(s-\sigma)) d \sigma\right] d s, \quad t \geq t_{0} .
$$

Using Tonelli's theorem, we reverse the order of integration and obtain

$$
=\int_{t}^{\infty} \frac{(s-t)^{n-1}}{(n-1) !}\left[\int_{c}^{d} q(s, \sigma) f(x(s-\sigma)) d \sigma\right] d s, \quad t \geq t_{0} .
$$

That is,

$$
x(t) \geq \frac{1}{r(t)} \int_{a}^{b} p(t, \tau) x(t-\tau) d \tau+\frac{1}{r(t)} \int_{t}^{\infty} \frac{(s-t)^{n-1}}{(n-1) !}\left[\int_{c}^{d} q(s, \sigma) f(x(s-\sigma)) d \sigma\right] d s, \quad t \geq t_{0} .
$$


Let $T \geq t_{0}$ be such that (2.6) hold, and $x(t-\mu)>0, t \geq T$. Now, we consider the set of functions

$$
\Omega=\left\{z \in C\left([T-\mu, \infty), R^{+}\right): 0 \leq z(t) \leq 1, t \geq T-\mu\right\}
$$

and define an operator $S$ on $\Omega$ as follows:

$$
(S z)(t)= \begin{cases}\frac{t-T+\mu}{\mu}(S z)(T)+\left(1-\frac{t-T+\mu}{\mu}\right), & T-\mu \leq t<T, \\ \frac{1}{x(t)}\left\{\frac{1}{r(t)} \int_{a}^{b} p(t, \tau) z(t-\tau) x(t-\tau) d \tau\right. & \\ \left.\quad+\frac{1}{r(t)} \int_{t}^{\infty} \frac{(s-t)^{n-1}}{(n-1) !}\left[\int_{c}^{d} q(s, \sigma) f(z(s-\sigma) x(s-\sigma)) d \sigma\right] d s\right\}, & t \geq T .\end{cases}
$$

Then, it follows from Lebesgue's dominated convergence theorem that $S$ is continuous. By using (2.6), it is easy to see that $S$ maps $\Omega$ into itself, and for any $z \in \Omega$, we have $(S z)(t)>0$, for $T-\mu \leq t<T$. Next, we define the sequence $z_{k}(t) \in \Omega$

$$
\begin{gathered}
z_{0}(t) \equiv 1, \quad t \geq T-\mu, \\
z_{k+1}(t)=\left(S z_{k}\right)(t), \quad t \geq T-\mu, \quad k=0,1, \ldots
\end{gathered}
$$

Then, by using (2.6) and a simple induction, we can easily see that

$$
0 \leq z_{k+1}(t) \leq z_{k}(t) \leq 1, \quad t \geq T-\mu .
$$

Set $\lim _{k \rightarrow \infty} z_{k}(t)=z(t), t \geq T-\mu$ then $z(t)$ satisfies

$$
z(t)= \begin{cases}\frac{t-T+\mu}{\mu}(S z)(T)+\left(1-\frac{t-T+\mu}{\mu}\right), & T-\mu \leq t<T, \\ \frac{1}{x(t)}\left\{\frac{1}{r(t)} \int_{a}^{b} p(t, \tau) z(t-\tau) x(t-\tau) d \tau\right. & \\ \left.\quad+\frac{1}{r(t)} \int_{t}^{\infty} \frac{(s-t)^{n-1}}{(n-1) !}\left[\int_{c}^{d} q(s, \sigma) f(z(s-\sigma) x(s-\sigma)) d \sigma\right] d s\right\}, & t \geq T .\end{cases}
$$

Again, set $\omega(t)=z(t) x(t)$, then $\omega(t)$ satisfies $\omega(t)>0, T-\mu \leq t \leq T$, and

$$
\omega(t)=\frac{1}{r(t)} \int_{a}^{b} p(t, \tau) \omega(t-\tau) d \tau+\frac{1}{r(t)} \int_{t}^{\infty} \frac{(s-t)^{n-1}}{(n-1) !}\left[\int_{c}^{d} q(s, \sigma) f(\omega(s-\sigma)) d \sigma\right] d s, \quad t \geq T .
$$

Thus, $\omega(t)$ is a positive solution of (1.1) for $t \geq T$. 
The following:

$$
\omega(t)>0, \quad t \geq T-\mu .
$$

Assume that there exists $t^{*} \geq T-\mu$, such that $\omega(t)>0$, for $T-\mu \leq t \leq t^{*}$, and $\omega\left(t^{*}\right)=0$. Then,

$$
\begin{aligned}
0=\omega\left(t^{*}\right)= & \frac{1}{r\left(t^{*}\right)} \int_{a}^{b} p\left(t^{*}, \tau\right) \omega\left(t^{*}-\tau\right) d \tau \\
& +\frac{1}{r\left(t^{*}\right)} \int_{t^{*}}^{\infty} \frac{\left(s-t^{*}\right)^{n-1}}{(n-1) !}\left[\int_{c}^{d} q(s, \sigma) f(\omega(s-\sigma)) d \sigma\right] d s, \quad t^{*} \geq T,
\end{aligned}
$$

which implies

$$
\begin{gathered}
p\left(t^{*}, \tau\right)=0, \\
q(s, \sigma) f(\omega(s-\sigma)) \equiv 0,
\end{gathered}
$$

which contradicts $\left(\mathrm{H}_{1}\right)$. Thus, $\omega(t)$ is an eventually bounded positive solution of (1.1).

The proof is complete.

Theorem 2.2. Assume all conditions of Lemma 1.3 hold, $n$ is a positive odd integer. And

$\left(\mathrm{H}_{1}\right) p(t, \tau)+q(t, \sigma)>0$, for sufficiently large $t$.

Then, (1.1) has an eventually positive solution if and only if inequality (1.2) has an eventually positive solution.

Proof. It is clear that an eventually positive solution of (1.1) is also an eventually positive solution of (1.2). So, it suffices to prove that if (1.2) has an eventually positive solution $x(t)$, for $t>t_{0}$, then so does (1.1). Set

$$
y(t)=r(t) x(t)-\int_{a}^{b} p(t, \tau) x(t-\tau) d \tau
$$

It follows from Lemma 1.3 and (1.2) that eventually, $y^{(n)}(t) \leq 0, y(t)>0$, which implies that there exists a nonnegative even integer $n^{*} \leq n-1$, such that eventually

$$
\begin{gathered}
y^{(k)}(t)>0, \quad\left(k=0,1, \ldots, n^{*}\right), \\
(-1)^{(k)} y^{(k)}(t)>0, \quad\left(k=n^{*}, \ldots, n-1\right) .
\end{gathered}
$$

We consider the following possible cases.

Case $1\left(n^{*}=0\right)$. Since $n$ is a positive integer, $n-1$ is an even integer, we can easily see that there exists a $T^{\prime}>0$, such $y^{(n-1)}(t)>0$, and $y^{n}(t) \leq 0, t \geq T^{\prime}, y^{(n-1)}(\infty) \geq 0$. 
By using (2.17) and integrating (1.2) from $t$ to $\infty$, we obtain

$$
\begin{aligned}
y^{(n-1)}(\infty)-y^{(n-1)}(t) & \leq-\int_{t}^{\infty}\left[\int_{c}^{d} q(s, \sigma) f(x(s-\sigma)) d \sigma\right] d s, \\
y^{(n-1)}(t) & \geq \int_{t}^{\infty}\left[\int_{c}^{d} q(s, \sigma) f(x(s-\sigma)) d \sigma\right] d s .
\end{aligned}
$$

By repeating the same procedure $n$ times and by using (2.31), we are led to the inequality:

$$
y(t) \geq \int_{t}^{\infty} d t_{n} \int_{t_{n}}^{\infty} d t_{n-1} \int_{t_{n-1}}^{\infty} d t_{n-2} \cdots \int_{t_{2}}^{\infty}\left[\int_{c}^{d} q(s, \sigma) f(x(s-\sigma)) d \sigma\right] d s, \quad t \geq t_{0} .
$$

Using Tonelli's theorem, we reverse the order of integration and obtain

$$
=\int_{t}^{\infty} \frac{(s-t)^{n-1}}{(n-1) !}\left[\int_{c}^{d} q(s, \sigma) f(x(s-\sigma)) d \sigma\right] d s, \quad t \geq t_{0} .
$$

That is,

$$
x(t) \geq \frac{1}{r(t)} \int_{a}^{b} p(t, \tau) x(t-\tau) d \tau+\frac{1}{r(t)} \int_{t}^{\infty} \frac{(s-t)^{n-1}}{(n-1) !}\left[\int_{c}^{d} q(t, \sigma) f(x(t-\sigma)) d \sigma\right] d s, \quad t \geq t_{0} .
$$

Let $T \geq t_{0}$ be such that (2.21) hold, and $x(t-\mu)>0, t \geq T$. Now, we consider the set of functions

$$
\Omega=\left\{z \in C\left([T-\mu, \infty), R^{+}\right): 0 \leq z(t) \leq 1, t \geq T-\mu\right\},
$$

and define an operator $S$ on $\Omega$ as follows:

$$
(S z)(t)= \begin{cases}\frac{t-T+\mu}{\mu}(S z)(T)+\left(1-\frac{t-T+\mu}{\mu}\right), & T-\mu \leq t<T, \\ \frac{1}{x(t)}\left\{\frac{1}{r(t)} \int_{a}^{b} p(t, \tau) z(t-\tau) x(t-\tau) d \tau\right. & \\ \left.\quad+\frac{1}{r(t)} \int_{t}^{\infty} \frac{(s-t)^{n-1}}{(n-1) !}\left[\int_{c}^{d} q(s, \sigma) f(z(s-\sigma) x(s-\sigma)) d \sigma\right] d s\right\}, & t \geq T .\end{cases}
$$


Then, it follows from Lebesgue's dominated convergence theorem that $S$ is continuous. By using (2.21), it is easy to see that $S$ maps $\Omega$ into itself, and for any $z \in \Omega$, we have $(S z)(t)>0$, for $T-\mu \leq t<T$. Next, we define the sequence $z_{k}(t) \in \Omega$

$$
\begin{gathered}
z_{0}(t) \equiv 1, \quad t \geq T-\mu, \\
z_{k+1}(t)=\left(S z_{k}\right)(t), \quad t \geq T-\mu, \quad k=0,1, \ldots .
\end{gathered}
$$

Then, by using (2.21) and a simple induction, we can easily see that

$$
0 \leq z_{k+1}(t) \leq z_{k}(t) \leq 1, \quad t \geq T-\mu
$$

Set $\lim _{k \rightarrow \infty} z_{k}(t)=z(t), t \geq T-\mu$, then $z(t)$ satisfies:

$$
z(t)= \begin{cases}\frac{t-T+\mu}{\mu}(S z)(T)+\left(1-\frac{t-T+\mu}{\mu}\right), & T-\mu \leq t<T, \\ \frac{1}{x(t)}\left\{\frac{1}{r(t)} \int_{a}^{b} p(t, \tau) z(t-\tau) x(t-\tau) d \tau\right. & \\ \left.\quad+\frac{1}{r(t)} \int_{t}^{\infty} \frac{(s-t)^{n-1}}{(n-1) !}\left[\int_{c}^{d} q(s, \sigma) f(z(s-\sigma) x(s-\sigma)) d \sigma\right] d s\right\}, & t \geq T .\end{cases}
$$

Again, set $\omega(t)=z(t) x(t)$, then $\omega(t)$ satisfies $\omega(t)>0, T-\mu \leq t \leq T$, and

$$
\omega(t)=\frac{1}{r(t)} \int_{a}^{b} p(t, \tau) \omega(t-\tau) d \tau+\frac{1}{r(t)} \int_{t}^{\infty} \frac{(s-t)^{n-1}}{(n-1) !}\left[\int_{c}^{d} q(s, \sigma) f(\omega(s-\sigma)) d \sigma\right] d s, \quad t \geq T
$$

Thus, $\omega(t)$ is a positive solution of (1.1) for $t \geq T$.

Consider the following:

$$
\omega(t)>0, \quad t \geq T-\mu .
$$

Assume that there exists $t^{*} \geq T-\mu$, such that $\omega(t)>0$, for $T-\mu \leq t \leq t^{*}$, and $\omega\left(t^{*}\right)=0$. Then,

$$
\begin{aligned}
0=\omega\left(t^{*}\right)= & \frac{1}{r\left(t^{*}\right)} \int_{a}^{b} p\left(t^{*}, \tau\right) \omega\left(t^{*}-\tau\right) d \tau \\
& +\frac{1}{r\left(t^{*}\right)} \int_{t^{*}}^{\infty} \frac{\left(s-t^{*}\right)^{n-1}}{(n-1) !}\left[\int_{c}^{d} q(s, \sigma) f(\omega(s-\sigma)) d \sigma\right] d s, \quad t^{*} \geq T,
\end{aligned}
$$


which implies

$$
\begin{gathered}
p\left(t^{*}, \tau\right)=0, \\
q(s, \sigma) f(\omega(s-\sigma)) \equiv 0,
\end{gathered}
$$

which contradicts $\left(\mathrm{H}_{1}\right)$. Thus, $\omega(t)$ is an eventually positive solution of equation.

Case $2\left(2 \leq n^{*} \leq n-1\right)$. By using (2.17) and integrating (1.2) from $t$ to $\infty$, we obtain

$$
y^{\left(n^{*}\right)}(t) \geq \int_{t}^{\infty} \frac{(s-t)^{n-1}}{(n-1) !}\left[\int_{c}^{d} q(s, \sigma) f(x(s-\sigma)) d \sigma\right] d s, \quad t \geq t_{0} .
$$

Let $T \geq 0$ be such that (2.17) and $\left(\mathrm{H}_{1}\right)$ hold. Integrating (2.31) from $T$ to $t$ and using (2.17), we have

$$
\begin{aligned}
x(t) \geq & \frac{1}{r(t)} \int_{a}^{b} p(t, \tau) x(t-\tau) d \tau \\
& +\frac{1}{r(t)} \int_{T}^{t} \frac{(t-s)^{n^{*}-1}}{\left(n^{*}-1\right) !} \int_{t}^{\infty} \frac{(u-s)^{n-n^{*}-1}}{\left(n-n^{*}-1\right) !}\left[\int_{c}^{d} q(s, \sigma) f(x(s-\sigma)) d \sigma\right] d u d s, \quad t \geq t_{0} .
\end{aligned}
$$

Using a method similar to the proof of Case 1 yields that (1.1) also has an eventually positive solution.

The proof is complete.

Theorem 2.3. Assume all conditions of Lemma 1.1 hold, $n$ is a positive even integer, and

$$
\left(\mathrm{H}_{1}\right) p(t, \tau)+q(t, \sigma)>0, \text { for sufficiently large } t .
$$

Then, (1.1) has an eventually positive solution if and only if inequality (1.2) has an eventually positive solution.

Proof. It is clear that an eventually bounded positive solution of (1.1) is also an eventually bounded positive solution of (1.2). So, it suffices to prove that if (1.2) has an eventually bounded positive solution $x(t)$, for $t>t_{0}$, then so does (1.1). Set

$$
y(t)=r(t) x(t)-\int_{a}^{b} p(t, \tau) x(t-\tau) d \tau
$$

It follows from Lemma 1.1 and (1.2) that eventually,

$$
\begin{gathered}
y^{(n)}(t) \leq 0,(-1)^{k} y^{(k)}(t)<0, \quad(k=0,1, \ldots, n-1), \\
\lim _{t \rightarrow \infty} y^{k}(t)=0, \quad(k=1,2, \ldots, n-1) .
\end{gathered}
$$


By using (1.9) and integrating (1.2) from $t$ to $\infty$, we obtain

$$
\begin{gathered}
y^{(n-1)}(\infty)-y^{(n-1)}(t) \leq-\int_{t}^{\infty}\left[\int_{c}^{d} q(s, \sigma) f(x(s-\sigma)) d \sigma\right] d s, \\
y^{(n-1)}(t) \geq \int_{t}^{\infty}\left[\int_{c}^{d} q(s, \sigma) f(x(s-\sigma)) d \sigma\right] d s .
\end{gathered}
$$

By repeating the same procedure $n$ times and by using (1.9), we are led to the inequality:

$$
y(t) \leq-\int_{t}^{\infty} d t_{n} \int_{t_{n}}^{\infty} d t_{n-1} \int_{t_{n-1}}^{\infty} d t_{n-2} \cdots \int_{t_{2}}^{\infty}\left[\int_{c}^{d} q(s, \sigma) f(x(s-\sigma)) d \sigma\right] d s, \quad t \geq t_{0} .
$$

Using Tonelli's theorem, we reverse the order of integration and obtain

$$
=-\int_{t}^{\infty} \frac{(s-t)^{n-1}}{(n-1) !}\left[\int_{c}^{d} q(s, \sigma) f(x(s-\sigma)) d \sigma\right] d s, \quad t \geq t_{0} .
$$

That is,

$$
x(t) \leq \frac{1}{r(t)} \int_{a}^{b} p(t, \tau) \omega(t-\tau) d \tau-\int_{t}^{\infty} \frac{(s-t)^{n-1}}{(n-1) !}\left[\int_{c}^{d} q(s, \sigma) f(x(s-\sigma)) d \sigma\right] d s, \quad t \geq t_{0} .
$$

Let $T \geq 0$ be such that (2.38) hold, and $x(t-\mu)>0, t \geq T$. Now, we consider the set of functions:

$$
\Omega=\{z \in C([T-\mu, \infty), R): 0<z(t) \leq 1, t \geq T-\mu\},
$$

and define an operator $S$ on $\Omega$ as follows:

$$
(S z)(t)=\left\{\begin{array}{c}
\frac{t-T+\mu}{\mu}(S z)(T)+\left(1-\frac{t-T+\mu}{\mu}\right), \\
T-\mu \leq t<T, \\
\frac{x(t)}{\frac{1}{r(t)} \int_{a}^{b} p(t, \tau) \frac{x(t-\tau)}{z(t-\tau)} d \tau-\frac{1}{r(t)} \int_{t}^{\infty} \frac{(s-t)^{n-1}}{(n-1) !}\left[\int_{c}^{d} q(s, \sigma) f\left(\frac{x(s-\sigma)}{z(s-\sigma)}\right) d \sigma\right] d s}, \\
t \geq T .
\end{array},\right.
$$


Then, it follows from Lebesgue's dominated convergence theorem that $S$ is continuous. By using (2.38), it is easy to see that $S$ maps $\Omega$ into itself and, for any $z \in \Omega$, we have $(S z)(t)<0$, for $T-\mu \leq t<T$. Next, we define the sequence $z_{k}(t) \in \Omega$ :

$$
\begin{gathered}
z_{0}(t) \equiv 1, \quad t \geq T-\mu, \\
z_{k+1}(t)=\left(S z_{k}\right)(t), \quad t \geq T-\mu, \quad k=0,1, \ldots
\end{gathered}
$$

Then, by using (2.38) and a simple induction, we can easily see that

$$
0<z_{k+1}(t) \leq z_{k}(t) \leq 1, \quad t \geq T-\mu .
$$

Set $\lim _{k \rightarrow \infty} z_{k}(t)=z(t), t \geq T-\mu$, then $z(t)$ satisfies

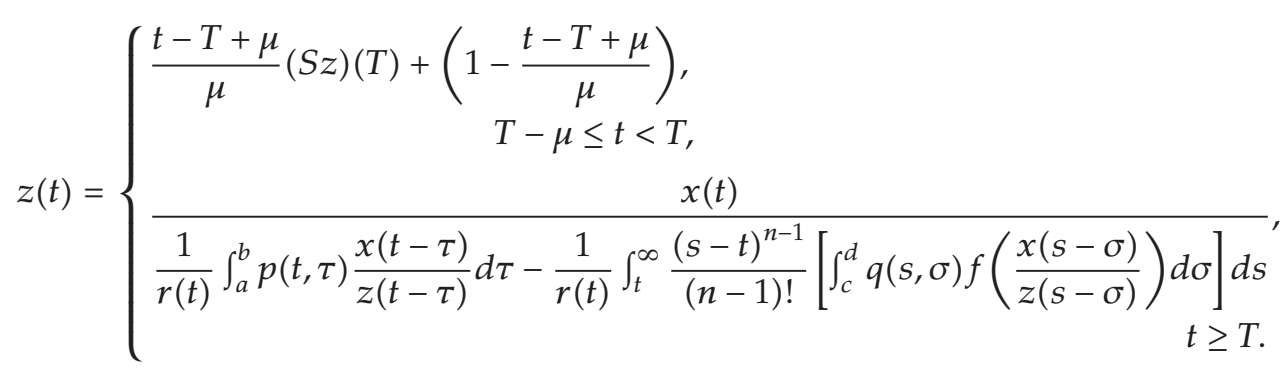

Again, set $\omega(t)=x(t) / z(t)$, then $\omega(t)$ satisfies $\omega(t)>0, T-\mu \leq t \leq T$, and

$$
\begin{aligned}
\omega(t)= & \frac{1}{r(t)} \int_{a}^{b} p(t, \tau) \omega(t-\tau) d \tau \\
& -\frac{1}{r(t)} \int_{t}^{\infty} \frac{(s-t)^{n-1}}{(n-1) !}\left[\int_{c}^{d} q(s, \sigma) f(\omega(s-\sigma)) d \sigma\right] d s, \quad t \geq T
\end{aligned}
$$

Thus, $\omega(t)$ is a positive solution of (1.1) for $t \geq T$.

Consider the following

$$
\omega(t)>0, \quad t \geq T-\mu .
$$

Assume that there exists $t^{*} \geq T-\mu$, such that $\omega(t)>0$, for $T-\mu \leq t \leq t^{*}$, and $\omega\left(t^{*}\right)=0$. Then,

$$
\begin{aligned}
0=\omega\left(t^{*}\right)= & \frac{1}{r\left(t^{*}\right)} \int_{a}^{b} p\left(t^{*}, \tau\right) \omega\left(t^{*}-\tau\right) d \tau \\
& -\frac{1}{r\left(t^{*}\right)} \int_{t^{*}}^{\infty} \frac{\left(s-t^{*}\right)^{n-1}}{(n-1) !}\left[\int_{c}^{d} q(s, \sigma) f(\omega(s-\sigma)) d \sigma\right] d s, \quad t^{*} \geq T,
\end{aligned}
$$


which implies

$$
\begin{gathered}
p\left(t^{*}, \tau\right)=0, \\
q(s, \sigma) f(\omega(s-\sigma)) \equiv 0,
\end{gathered}
$$

which contradicts $\left(\mathrm{H}_{1}\right)$. Thus, $\omega(t)$ is an eventually positive solution of (1.1).

The proof is complete.

Example 2.4. Consider high-order neutral differential equation with distributed delay

$$
\left(x(t)-\int_{1}^{2} e^{-t} x(t-\tau) d \tau\right)^{(n)}+\frac{1}{2 e^{-1}-3 e^{-2}} \int_{1}^{2} \sigma x(t-\sigma) d \sigma=0
$$

Here, $r(t) \equiv 1, p(t-\tau)=e^{-t}, q(t, \sigma)=\sigma / 2 e^{-1}-3 e^{-2}, a=c=1, b=d=2$. It is easy to see that

$$
r(t) \equiv 1<\infty, \quad \frac{\int_{1}^{2} e^{-t} d \tau}{1}=e^{-t}<1, \quad t>0
$$

From Theorem 2.2, we have that (1.1) has an eventually positive solution if and only if inequality (1.2) has an eventually positive solution. In fact, $x(t)=e^{t}$ is a positive solution of (1.1).

\section{Acknowledgments}

This research is supported by the Natural Sciences Foundation of Shanxi Province and Scientific Research Project of Shanxi Datong University. The authors are greatly indebted to the referees for their valuable suggestions and comments.

\section{References}

[1] Y. Liu, H. Zhao, and J. Yan, "Eventually positive and bounded solutions of even-order nonlinear neutral differential equations," Applied Mathematics Letters, vol. 21, no. 11, pp. 1118-1123, 2008.

[2] Z. Ouyang, Y. Li, and M. Qing, "Eventually positive solutions of odd-order neutral differential equations," Applied Mathematics Letters, vol. 17, no. 2, pp. 159-166, 2004.

[3] I. Györi and G. Ladas, Oscillation Theory of Delay Differential Equations With Applications, Clarendon Presss, Oxford, UK, 1991.

[4] T. Candan and R. S. Dahiya, "On the oscillation of certain mixed neutral equations," Applied Mathematics Letters, vol. 21, no. 3, pp. 222-226, 2008.

[5] T. Candan and R. S. Dahiya, "Existence of nonoscillatory solutions of first and second order neutral differential equations with distributed deviating arguments," Journal of the Franklin Institute, vol. 347, no. 7, pp. 1309-1316, 2010.

[6] J. G. Dong, “Oscillation of solutions for first order neutral differential equations with distributed deviating arguments," Computers and Mathematics with Applications, vol. 58, no. 4, pp. 784-790, 2009.

[7] X. Lin, W. Liu, and Y. Yu, "Oscillation criteria for even-order half-linear distributed delay differential equations with damping," Computers and Mathematics with Applications, vol. 60, no. 8, pp. 2206-2211, 2010.

[8] L. Berezansky and E. Braverman, "Oscillation of equations with an infinite distributed delay," Computers and Mathematics with Applications, vol. 60, no. 9, pp. 2583-2593, 2010. 


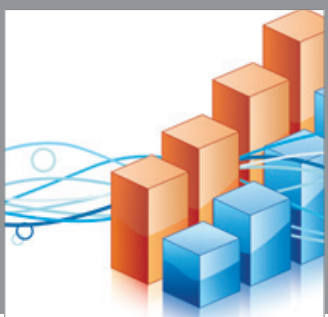

Advances in

Operations Research

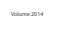

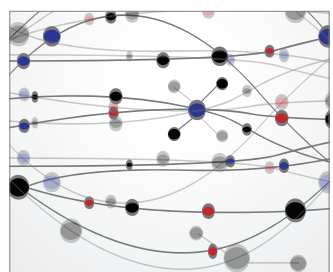

\section{The Scientific} World Journal
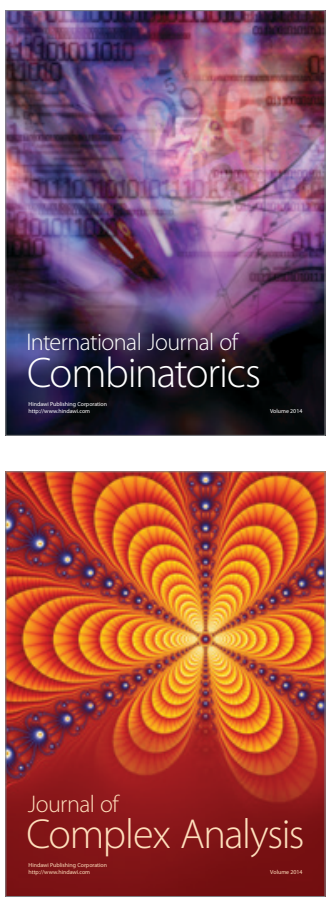

International Journal of

Mathematics and

Mathematical

Sciences
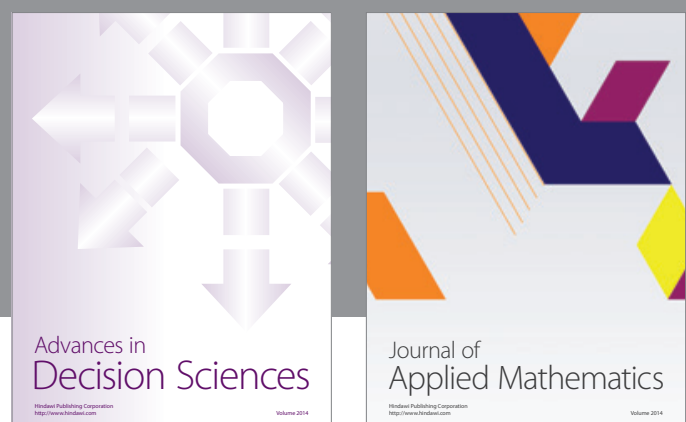

Journal of

Applied Mathematics
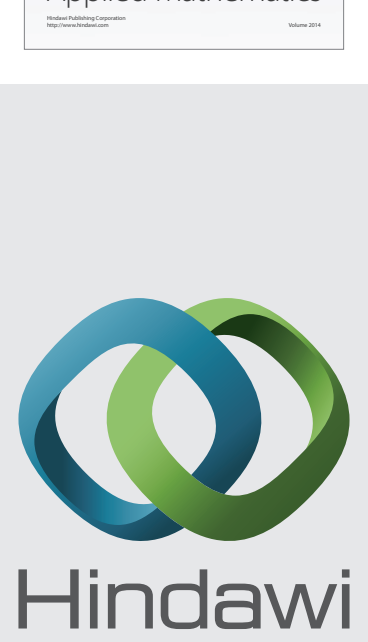

Submit your manuscripts at http://www.hindawi.com
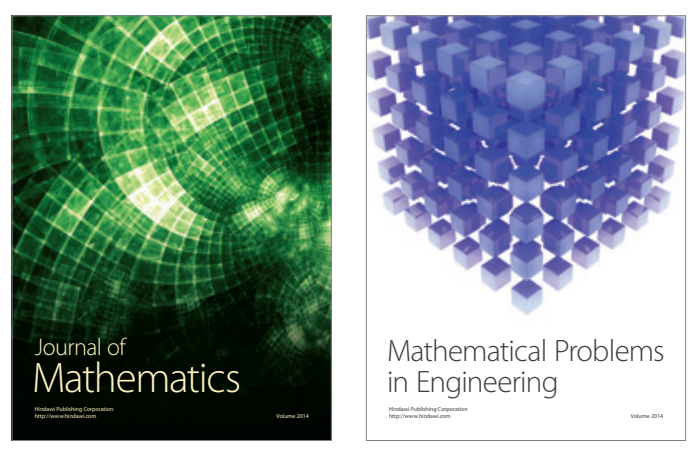

Mathematical Problems in Engineering
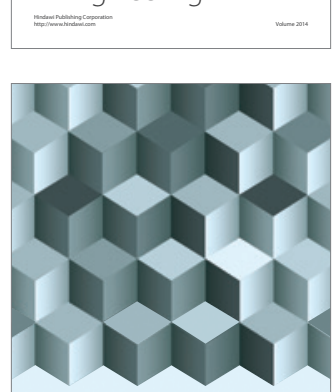

Journal of

Function Spaces
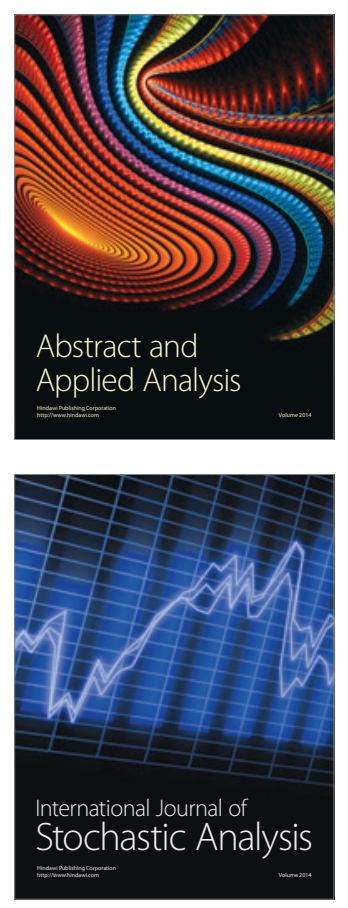

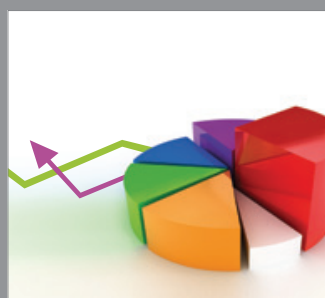

ournal of

Probability and Statistics

Promensencen
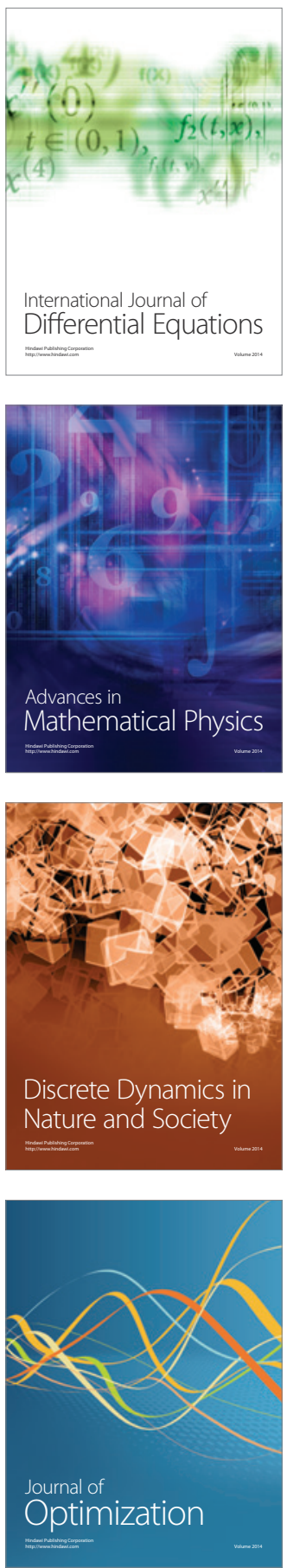